\title{
$\underline{\mathbf{P}-163}$
}

\section{Antioxidant and Alpha-Glucosidase Activities of Phyllanthus Niruri Extracted with Different Solvents}

\author{
Ahmed Mediani ${ }^{\mathrm{a},}$, Faridah Abas ${ }^{\mathrm{a}, \mathrm{b}}$, Alfi Khatib ${ }^{\mathrm{a}, \mathrm{b}}$, Intan Safinar Ismail ${ }^{\mathrm{b}}$, Khozirah Shaari ${ }^{\mathrm{b}}$, Amin \\ Ismail $^{\mathrm{c}}$ and N.H. Lajis ${ }^{\mathrm{d}}$ \\ ${ }^{a}$ Department of Food Science, Faculty of Food Science and Technology, Universiti Putra Malaysia, 43400 Serdang, \\ Selangor, Malaysia; ${ }^{b}$ Laboratory of Natural Products, Institute of Bioscience, Universiti Putra Malaysia, 43400 Serdang \\ Selangor, Malaysia; ${ }^{c}$ Department of Nutrition and Dietetics, Faculty of Medicine and Health Sciences, Universiti Putra \\ Malaysia, 43400 Serdang Selangor, Malaysia; ${ }^{d}$ Scientific Chair for Prophetic Medicine Unit, College of Medicine, Taibah \\ University, P.O. Box 30001, al-Madinah al-Munawarah, Saudi Arabia; E-mail: mediani82@yahoo.com
}

Several studies recommended that plants are potential source of bioactive compounds. Phyllanthus niruri is one of plants, which used as a traditional medicine due its richness of phytomedicine metabolites. Identifying the appropriate solvent of extracting these bioactive components from Phyllanthus niruri is thus a crucial step to increase its significance and competence. The influence of drying on its quality and productivity was also evaluated. Three extracting solvents (ethanol, methanol, water) with different ratio were assessed for their effects on biological activities of $P$. niruri which are dried with three drying methods (air, oven and freeze). The antioxidant activity was evaluated using free radical scavenging ability (DPPH), FRAP and total phenolic content (TPC) assays and antidiabetic by $\alpha$-glucosidase assay. The selectivity of solvents was also evaluated in terms of the phytochemical compounds present and their concentrations based on quantifying them by LCMS. As function to solvent and drying methods, freeze dried, $P$. niruri extracted with $80 \%$ methanol and water exhibited valuable and higher antioxidant and antidiabetic activities. Principal component analysis (PCA) revealed the relationship among the potential antioxidant activity, total phenolic, $\alpha$-glucosidase and metabolites levels of the extracts. The present study proposes that $P$. niruri can be a prominent and constructive medicinal plant. 